Abanico Veterinario. Enero-Diciembre 2020; 10:1-15. http://dx.doi.org/10.21929/abavet2020.16 Nota Corta. Recibido: 20/03/2020. Aceptado: 02/07/2020. Publicado: 16/07/2020.

\title{
Respuesta serológica contra Mannheimia haemolytica y su leucotoxina en conejos suplementados con selenio
}

Serological response against Mannheimia haemolytica and its leukotoxin in rabbits supplemented with selenium

\section{Díaz-Sánchez Víctor ${ }^{1 *} \mathrm{ID}$, Ciriaco-Solano Lina ${ }^{1 \mathrm{ID}}$, Rodríguez-Patiño Gabriela ${ }^{1 \mathrm{ID}}$, López-Arellano Raquel ${ }^{1 \mathrm{ID}}$, Revilla-Vázquez Alma ${ }^{1 \mathrm{ID}}$, Morales-Álvarez José ${ }^{2 \mathrm{ID}}$}

${ }^{1}$ Universidad Nacional Autónoma de México, Facultad de Estudios Superiores Cuautitlán. Ciudad de México, México. ${ }^{2}$ Instituto Nacional de Investigaciones Forestales Agrícolas y Pecuarias. Ciudad de México, México. *Autor responsable y de correspondencia: Díaz-Sánchez Víctor, Universidad Nacional Autónoma de México, Facultad de Estudios Superiores Cuautitlán, Cuautitlán Izcalli, Estado de México, México, C.P. 54714. victorm_diazs@comunidad.unam.mx, lecs19943@gmail.com, grp2284@yahoo.com.mx, rlajjd@yahoo.com.mx, revillalma@gmail.com,morales62@yahoo.com

\section{RESUMEN}

La selenodeficiencia tiene un impacto negativo en la respuesta inmune de los animales. El objetivo del trabajo fue evaluar la suplementación con selenio y su efecto sobre la respuesta contra Mannheimia haemolytica y su leucotoxina. Se utilizaron 21 conejos; éstos fueron distribuidos en tres grupos $(n=7) ; A$ : se les administró selenio más una bacterina-toxoide; B: se les administró bacterina-toxoide. C: considerado control. Se estimó el contenido de selenio en sangre por espectrofotometría de absorción atómica. Se evaluó la respuesta a los antígenos a través una ELISA. Se realizó un análisis de varianza y de Tukey para determinar la significancia estadística, considerando un valor $\mathrm{P}<0.05$. En la cuantificación de selenio se observó una diferencia entre $A$ con respecto a $B$ y $C(P<0.05)$. En la evaluación de $\lg G$ contra $M$. haemolytica, hubo una diferencia entre $A$ con respecto a $B$ y $C(P<0.05)$. Para IgG contra la leucotoxina, no se observaron diferencias entre $A$ y $B(P>0.05)$, pero si de estos con respecto a $C(P<0.05)$. En conclusión, los animales suplementados tuvieron mayores concentraciones de selenio. Ésta tuvo efectos positivos sobre la respuesta contra $M$. haemolytica, sin embargo, no se encontraron diferencias para la respuesta contra la leucotoxina.

Palabras clave: Mannheimia haemolytica, selenio, conejos e inmunidad.

\begin{abstract}
Selenodeficiency has a negative impact on the immune response of animals. The objective of the work was to evaluate selenium supplementation and its effect on the response against Mannheimia haemolytica and its leukotoxin. 21 rabbits were used, these were distributed in three groups $(n=7)$; A: Selenium plus bacterintoxoid was administered; B: the bacterin-toxoid was administered. C: considered control. Blood selenium content was estimated by atomic absorption spectrophotometry. The response to antigens was evaluated through an ELISA. An analysis of variance and Tukey were performed to determine statistical significance, considering a $P$ value $<0.05$. In selenium quantification, a difference was observed between $A$ with respect to $B$ and $C(P<0.05)$. In the evaluation of $\lg G$ against $M$. haemolytica, there was a difference between $A$ with respect to $B$ and $C(P<0.05)$. For Ig $G$ against leukotoxin, no differences were observed between $A$ and $B(P>0.05)$, but of these with respect to $C(P<0.05)$. In conclusion, the supplemented animals had higher concentrations of selenium. This had positive effects on the response against $M$. haemolytica, however, no differences were found for the response against leukotoxin.
\end{abstract}

Keywords: Mannheimia haemolytica, selenium, rabbits, immunity. 


\section{INTRODUCCIÓN}

Mannheimia haemolytica es una bacteria habitante normal del tracto respiratorio superior de rumiantes, la cual puede llegar a causar problemas neumónicos asociados a una inmunosupresión, lo cual puede llevar a la muerte de los animales (De la Rosa et al., 2012). Se ha observado, que la deficiencia de algunos minerales en los animales tiene como consecuencia un efecto negativo en la respuesta inmunológica frente a la presencia de antígenos (Radwinska y Zarczynska, 2014). Es por esto que existe un interés en el uso de suplementos que nivelen los requerimientos de minerales, para mejorar el estatus inmunológico y los procesos productivos de los animales (Campos, 2015).

Actualmente se utilizan premezclas o soluciones parenterales para evitar la carencia de minerales como el selenio, el cual es un micronutriente esencial para los mamíferos, necesario en los procesos de crecimiento, producción y reproducción de los animales (Mehdi y Dufrasne, 2016). A través de las selenoproteínas tienen funciones antioxidantes, y de esta forma participa en el correcto funcionamiento de órganos, como: corazón, hígado, riñones, páncreas, testículos y tiroides (Ghany y Tórtora-Pérez, 2010).

Se ha observado que la deficiencia de este mineral afecta la capacidad de los neutrófilos y macrófagos, para fagocitar y destruir a los antígenos; además de que la vida de estas células se ve disminuida, afectándose los fenómenos de presentación antigénica y la posterior producción de inmunoglobulinas en sangre; factor que determina mayor prevalencia y severidad de las enfermedades (Avery y Hoffmann, 2018).

La suplementación con selenio puede mejorar la respuesta inmune en diferentes padecimientos de los animales. La concentración de anticuerpos se eleva en animales suplementados con selenio, frente al desafío con diferentes antígenos; en comparación con los animales que no fueron suplementados (Gelderman y Clapper, 2013).

El objetivo del presente trabajo fue evaluar mediante una ELISA indirecta la suplementación con selenio por vía parenteral, y la respuesta de anticuerpos contra Mannheimia haemolytica serotipo A2 y su leucotoxina, utilizando a conejos como modelo biológico. La hipótesis de este trabajo, es que, si se suplementa a conejos con selenio de forma parenteral, éstos tendrán una mayor respuesta serológica a los antígenos de Mannheimia haemolytica serotipo A2 y su leucotoxina, a diferencia de los animales que no fueron suplementados.

\section{MATERIAL Y MÉTODOS}

\section{Características de las unidades experimentales, distribución de grupos y administración de tratamiento}

Se utilizaron 21 conejos, raza Nueva Zelanda, con un peso promedio de $3.2 \mathrm{~kg}$ y 6 meses de edad respectivamente, como modelo biológico. Los animales fueron manejados bajo las normas de cuidado del Instituto Nacional de Investigaciones Forestales, Agrícolas y Pesqueras, en el Centro Nacional de Investigaciones Disciplinarias en Microbiología Animal, con fundamento en la Ley Federal de Salud Animal (título tercero. Capítulo I del bienestar de los animales) y en la norma oficial mexicana NOM-062-ZOO-1999 
(numerales 4.2.2; 4.2.2.1; 4.2.2.2; 4.2.2.3). Los animales fueron alojados en jaulas individuales de acero inoxidable de $50 \mathrm{~cm} \times 30 \mathrm{~cm}$, mantenidos con pellets de alfalfa y agua ad libitum; distribuidos aleatoriamente en tres grupos; grupo $A$ : $(n=7)$, se les administró una solución de selenio ( $10.95 \mathrm{mg}$ selenito de sodio por cada $\mathrm{mL}$ de solución), a una dosis de $0.25 \mathrm{mg} / \mathrm{Kg}$ de peso vivo más $2 \mathrm{~mL}$ de una bacterina-toxoide, la cual contenía una suspensión bacteriana con $1 \times 10^{6}$ unidades formadoras de colonias por mililitro (UFC/mL), a base de Mannheimia haemolytica serotipo A2 con leucotoxoide de la bacteria por vía subcutánea; grupo B: $(n=7)$, se les administró $2 \mathrm{~mL}$ de la bacterinatoxoide vía subcutánea, y grupo C: $(\mathrm{n}=7)$, se les administró $2 \mathrm{~mL}$ de solución salina fisiológica al 10\% por vía subcutánea. Este grupo fue considerado el grupo control. Los tratamientos se administraron en la semana 0 y la semana 2 para todos los animales dentro del estudio.

\section{Toma de muestras y procesamiento}

Las muestras se tomaron de forma semanal. Se obtuvo sangre de todos los grupos experimentales de la vena marginal auricular, utilizando el sistema Vacutainer ${ }^{\circledR}$; se tomó $1 \mathrm{~mL}$ de sangre en un tubo neutro sin anticoagulante y $1 \mathrm{~mL}$ en un tubo con heparina. Los tubos neutros fueron centrifugados a $4,500 \mathrm{~g}$ durante $5 \mathrm{~min}$ para obtener el suero.

\section{Pruebas de laboratorio}

Las pruebas en sangre y suero se realizaron de la siguiente manera; para la estimación del contenido de selenio en sangre se utilizó el método de espectrofotometría de absorción atómica con generador de hidruros, siguiendo el método descrito por GhanyHefnawy et al., 2007. Para la obtención de los antígenos para las pruebas de ELISA, se sonicó a Mannheimia haemolytica serotipo A2, para exponer sus antígenos, siguiendo la técnica descrita por Solanet et al., 2011. En la obtención de la leucotoxina de Mannheimia haemolytica se siguió el método descrito por Morales-Álvarez et al., 1993.

Para la evaluación de la respuesta a Mannheimia haemolytica y a su leucotoxina se realizó una prueba de ELISA indirecta, esta prueba se realizó en microplacas de polietileno de 96 pozos de fondo plano, se agregaron $100 \mu \mathrm{L}$ de cada uno de los antígenos en una dilución 1:20 en cada pozo por triplicado, incubando durante 24 horas a $37^{\circ} \mathrm{C}$. Pasado este tiempo se realizó un ciclo de tres lavados con PBS Tween-20 mediante un lavador de microplacas; luego se agregó una solución de leche descremada al $2 \%$ en PBS, con la finalidad de ocupar sitios en donde no hubo adsorción del antígeno, dejándola por 60 min a $37^{\circ} \mathrm{C}$. Posteriormente se realizaron 3 lavados con PBS-Tween 20. Por último se cubrieron las placas y se almacenaron a $4^{\circ} \mathrm{C}$, hasta su uso. Posteriormente se depositaron $100 \mu \mathrm{L}$ de los sueros problema en cada pozo, a una dilución de 1:20 en PBS, incubando a $37^{\circ} \mathrm{C}$, en una estufa bacteriológica por espacio de 60 min. Pasado este tiempo se realizaron tres lavados con PBS Tween-20; se agregaron $100 \mu \mathrm{L}$ de conjugado conejo anti-ovino IgG en cada pozo, a una dilución de 1:2000 en PBS, incubando 60 min a $37^{\circ} \mathrm{C}$. Posteriormente se realizaron tres lavados con PBS Tween-20 y se adicionaron $100 \mu \mathrm{L}$ de sustrato (ABTS de Sigma Chemicals Co). 
Finalmente se realizó la lectura en un espectrofómetro múltiple, calibrado a 405 nm (EIA multi-well reader de Sigma D).

\section{Análisis estadístico}

Se realizó un análisis de varianza para determinar la significancia estadística de cada una de las variables a medir; se consideró un valor de $\mathrm{P}<0.05$ para determinar la significancia de los datos. Posteriormente, para identificar entre cuales grupos hubo una diferencia significativa, se utilizó la prueba estadística de Tukey; para ello se calculó la diferencia honestamente significativa o HSD (Honestly significant difference) para cada una de las variables a medir. Se consideró como variables independientes a la dosis de selenio, dosis de la bacterina-toxoide y el tiempo de muestreo. Como variables dependientes se consideraron los niveles de selenio en sangre y absorbancias en suero para los antígenos evaluados. Para el análisis se utilizó el programa Statgraphics Centurion 16.1.11.

\section{RESULTADOS}

\section{Cuantificación de selenio en sangre de los grupos experimentales}

Se realizó la evaluación de la cuantificación de selenio en sangre en los grupos experimentales. Para determinar diferencias significativas se realizó un análisis de varianza, considerando un valor de $\mathrm{P}<0.05$ (tabla 1).

Tabla 1. Análisis de varianza de la cuantificación de selenio en sangre de los grupos experimentales

\begin{tabular}{l|c|c|c|c|c}
\hline \multicolumn{1}{c|}{ Fuente } & Suma de cuadrados & Grados de libertad. & Promedio de los cuadrados & Valor $\mathbf{f}$ & Valor $\mathbf{p}$ \\
\hline Entre grupos & 0.007 & 2 & 0.004 & 4.990 & 0.026 \\
Dentro de los grupos & 0.009 & 12 & 0.001 & \\
Total & 0.0158 & 14 & & \\
\hline
\end{tabular}

En la tabla 1, se observa el análisis de varianza de los niveles de selenio en sangre de los grupos experimentales; se obtuvo un valor $\mathrm{P}<0.05$, por lo que existe una relación estadísticamente significativa entre las concentraciones de selenio en sangre entre grupos experimentales. Estas diferencias se pueden observar en la figura 1.

En la figura 1, se observan las medias y errores estándar para la cuantificación de selenio en sangre de los grupos experimentales. El grupo A presenta una concentración promedio mayor, a diferencia de los grupos $\mathrm{B}$ y $\mathrm{C}(\mathrm{P}<0.05)$. Durante el experimento, estos últimos sin diferencia estadística entre ellos $(P>0.05)$. Para corroborarlo se realizó una prueba de Tukey, se calculó el HSD, obteniendo un valor de $0.045 \mu \mathrm{g} / \mathrm{g}$. Al compararlo con el valor obtenido de restar las medias de los grupos, se obtuvo una diferencia estadísticamente significativa entre el grupo A, con respecto al grupo B y el grupo C. 


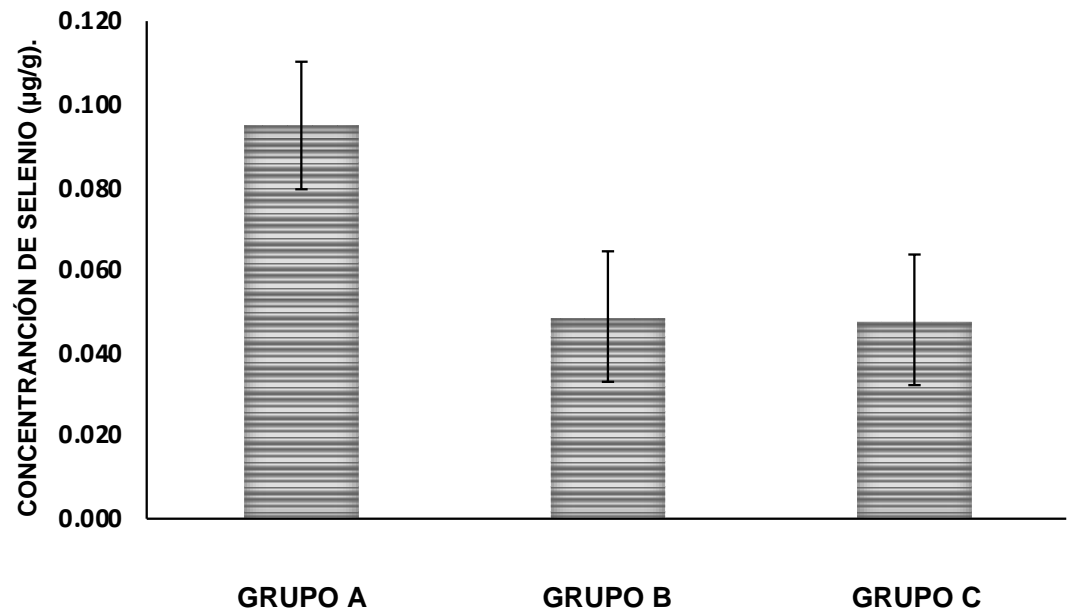

Figura 1. Medias y errores estándar de la cuantificación de selenio en sangre de los grupos experimentales

A continuación, se muestran las concentraciones semanales de selenio en sangre para los grupos de estudio (figura 2). Se observa la cuantificación semanal de selenio en sangre para los grupos experimentales. Se observa que en las semanas 0,2 y 3 , el grupo A presenta concentraciones de selenio en sangre mayores, en comparación con los grupos $B$ y $C(P<0.05)$; estos últimos sin diferencia significativa entre ellos $(P>0.05)$, para estas semanas. Por el contrario, en las semanas 1 y 4 los tres grupos no presentan diferencias significativas en las concentraciones de selenio en sangre $(P>0.05)$.

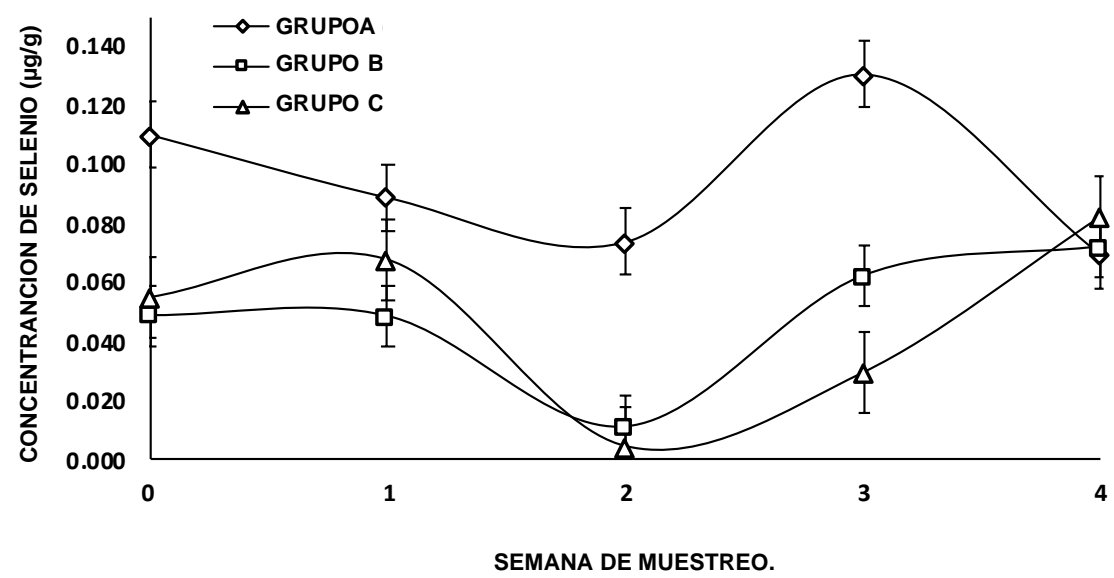

Figura 2. Cuantificación de selenio semanal en sangre de los grupos experimentales

Evaluación de la respuesta humoral contra Mannheimia haemolytica serotipo A2 en suero de los grupos experimentales

Se realizó la evaluación de las absorbancias a $405 \mathrm{~nm}$ para lgG en los sueros de los grupos experimentales, contra los antígenos de Mannhemia haemolytica. Se realizó un 
análisis de varianza para determinar la significancia estadística entre grupos, para lo cual se consideró un valor de $\mathrm{P}<0.05$ (tabla 2 ).

Tabla 2. Análisis de varianza de las absorbancias obtenidas a $405 \mathrm{~nm}$ para IgG en suero de los grupos experimentales contra los antígenos de Mannheimia haemolytica

\begin{tabular}{l|c|c|c|c|c}
\hline \multicolumn{1}{c|}{ Fuente } & $\begin{array}{c}\text { Suma de } \\
\text { cuadrados }\end{array}$ & $\begin{array}{c}\text { Grados de } \\
\text { libertad. }\end{array}$ & $\begin{array}{c}\text { Promedio de los } \\
\text { cuadrados }\end{array}$ & Valor F & Valor P \\
\hline Entre grupos & 0.308 & 2 & 0.154 & 4.941 & 0.027 \\
Dentro de los & 0.375 & 12 & 0.031 & & \\
grupos & 0.683 & 14 & & & \\
Total & & & & \\
\hline
\end{tabular}

En la tabla 2, se observa el análisis de varianza para las absorbancias obtenidas a 405 $\mathrm{nm}$ para lgG en los sueros de los grupos experimentales, contra los antígenos de Mannhemia haemolytica. Se observa un valor $\mathrm{P}<0.05$, por lo que existe una relación estadísticamente significativa para las absorbancias para lgG entre los grupos de estudio. Estas diferencias se pueden observar en la figura 3.

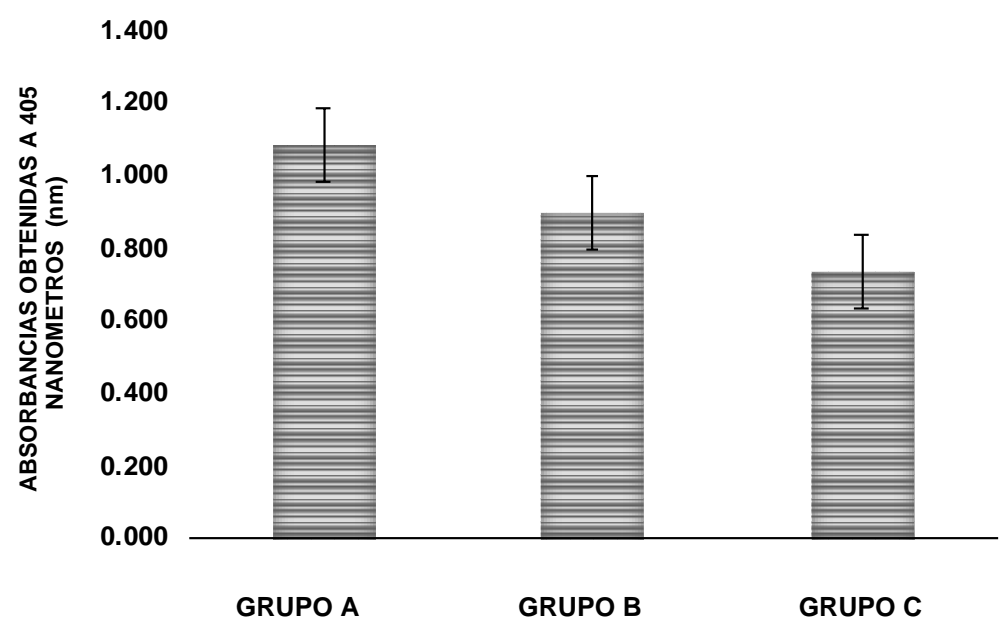

Figura 3. Medias y errores estándar de las absorbancias para IgG en suero de los grupos experimentales contra Mannheimia haemolytica

En la figura 3, se observan las medias y los errores estándar para las absorbancias obtenidas a $405 \mathrm{~nm}$ para IgG en los sueros de los grupos experimentales, contra los antígenos de Mannhemia haemolytica. El Grupo A tuvo absorbancias mayores a diferencia de los grupos $\mathrm{B}$ y $\mathrm{C}(\mathrm{P}<0.05)$. Durante el experimento, estos últimos sin diferencia estadística significativa $(P>0.05)$. Se realizó una prueba de Tukey, se calculó el HSD, obteniendo un valor de 0.272. Al compararlo con el valor obtenido de restar las medias de los grupos, se observó una diferencia estadísticamente significativa entre el grupo $A$, con respecto al grupo $B$ y el grupo $C$.

A continuación, se muestran las absorbancias semanales obtenidas a $405 \mathrm{~nm}$ para lgG, contra Mannheimia haemolytica para los grupos de estudio (figura 4). 


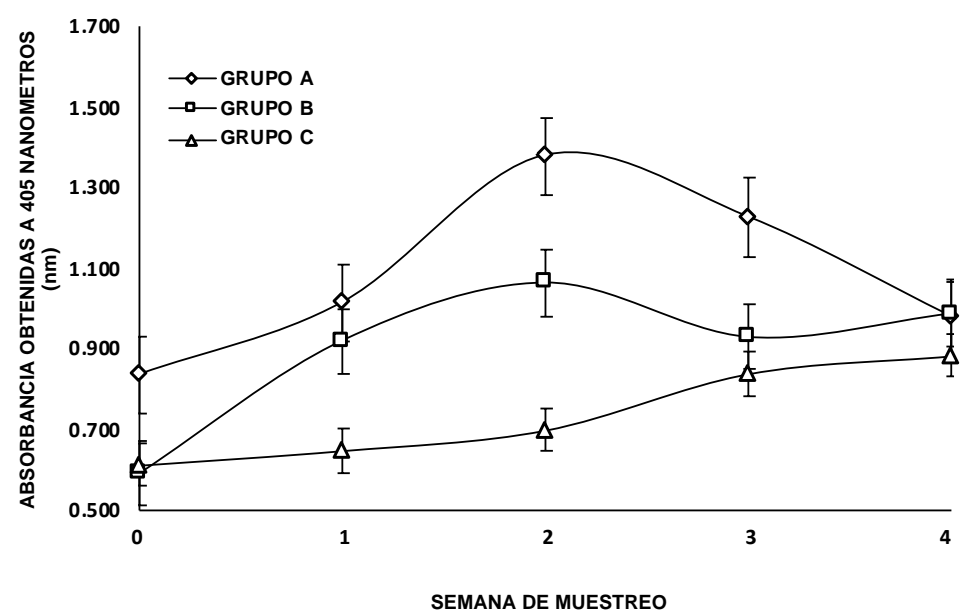

Figura 4. Absorbancias semanales para IgG de los sueros de los grupos experimentales, contra Mannheimia haemolytica

En la figura 4, se observan las absorbancias semanales obtenidas a $405 \mathrm{~nm}$ para lgG, contra Mannhemia haemolytica en los grupos de estudio. En las semanas 0, 2 y 3, se observa que el grupo A presenta mayores absorbancias, en comparación con los grupos $B$ y $C(P<0.05)$. En las semanas 1 y 4 no se observan diferencias entre $A$ y $B$, y entre $A$, $B$ y $C$, respectivamente $(P>0.05)$.

\section{Evaluación de la respuesta humoral a la leucotoxina de Mannheimia haemolytica en suero de los grupos experimentales}

Se evaluaron las absorbancias obtenidas a $405 \mathrm{~nm}$ para lgG en suero de los grupos experimentales, contra la leucotoxina de Mannhemia haemolytica. Se realizó un análisis de varianza para determinar diferencias significancias, considerando un valor $\mathrm{P}<0.05$ (tabla 3).

Tabla 3. Análisis de varianza de las absorbancias obtenidas a $405 \mathrm{~nm}$ para la leucotoxina de Mannheimia haemolytica de los sueros de los grupos experimentales

\begin{tabular}{l|c|c|c|c|c}
\hline \multicolumn{1}{c|}{ Fuente } & $\begin{array}{c}\text { Suma de } \\
\text { cuadrados }\end{array}$ & $\begin{array}{c}\text { Grados de } \\
\text { libertad. }\end{array}$ & $\begin{array}{c}\text { Promedio de los } \\
\text { cuadrados }\end{array}$ & Valor F & Valor P \\
\hline Entre grupos & 0.827 & 2 & 0.414 & 4.026 & 0.046 \\
Dentro de los & 1.233 & 12 & 0.103 & & \\
grupos & 2.060 & 14 & & & \\
Total & & & & \\
\hline
\end{tabular}

En la tabla 3 se observa el análisis de varianza para las absorbancias obtenidas a 405 $\mathrm{nm}$ para IgG en suero de los grupos experimentales, contra la leucotoxina de Mannhemia haemolytica. Se observa un valor $\mathrm{P}<0.05$, por lo que existe una relación estadísticamente significativa para las absorbancias para IgG entre los grupos de estudio. Estas diferencias se pueden observar en la figura 5. 


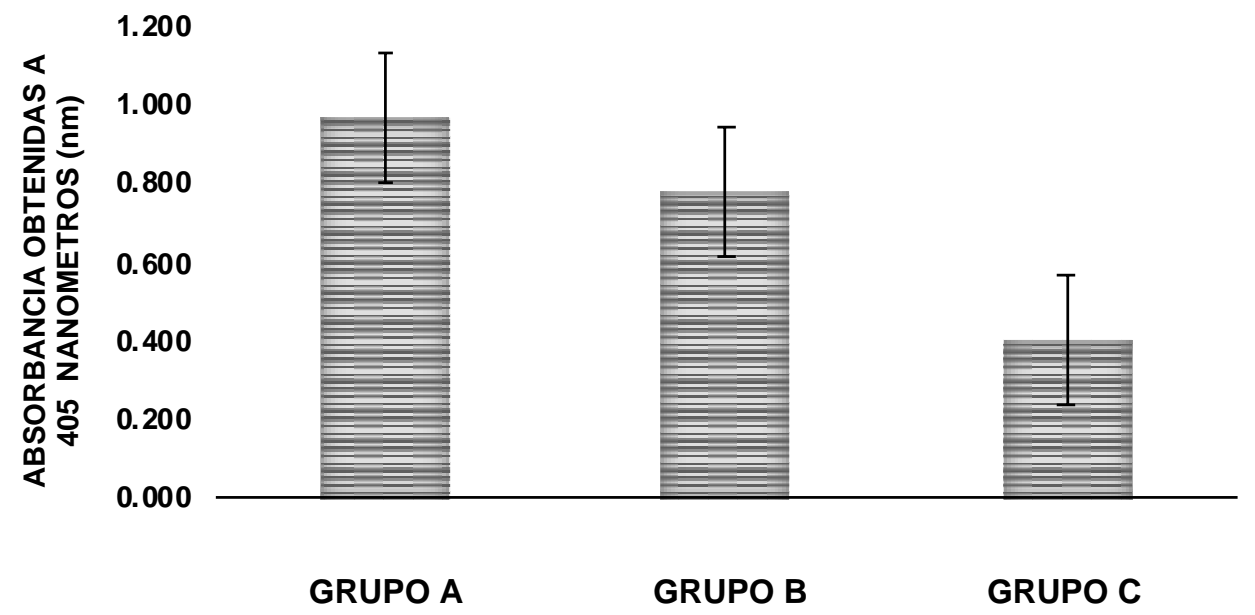

Figura 5. Medias de absorbancias para IgG en suero de los grupos experimentales de la leucotoxina de Mannheimia haemolytica serotipo A2 en suero

En la figura 5 se observan las medias y los errores estándar para las absorbancias obtenidas a $405 \mathrm{~nm}$ para IgG en suero de los grupos experimentales, contra la leucotoxina de Mannhemia haemolytica. Los grupos A y B no presentan diferencias significativas entre ellos ( $P>0.05)$; sin embargo, presentan mayores absorbancias que el grupo $C(P<0.05)$ durante el experimento. Se realizó la prueba de Tukey, se calculó el HSD, obteniendo un valor de 0.540. Al compararlo con el valor obtenido de restar las medias de los grupos, se observa una diferencia estadísticamente significativa entre el grupo A, con respecto al grupo C; el cual no fue desafiado con la leucotoxina.

A continuación, se muestran las absorbancias semanales obtenidas a $405 \mathrm{~nm}$ para lgG contra la leucotoxina para los grupos de estudio (figura 6).

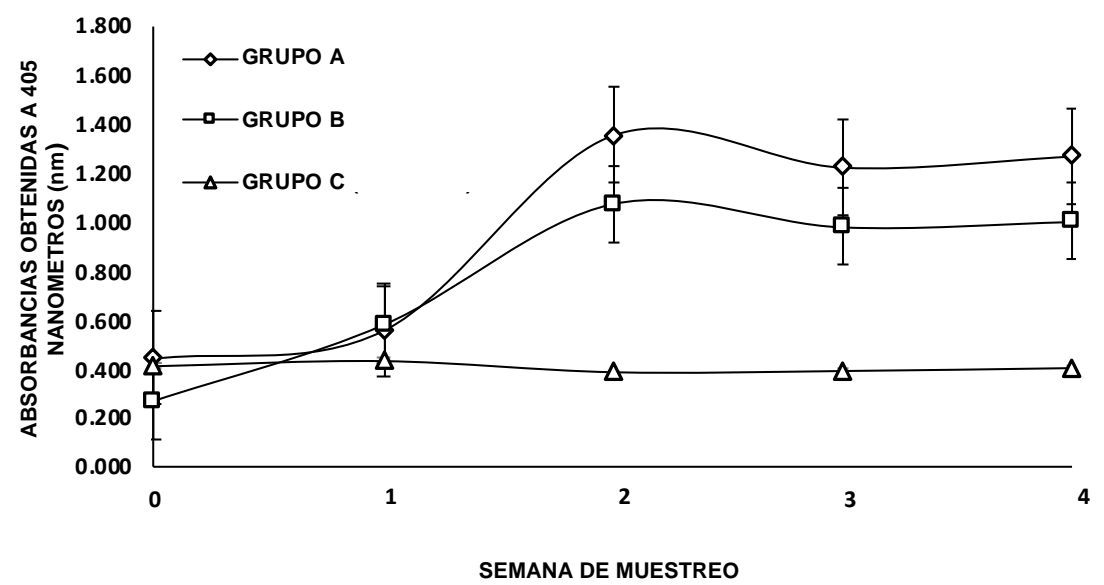

Figura 6. Promedio semanal de las absorbancias para IgG en los sueros de los grupos experimentales, contra la leucotoxina de Mannhemia haemolytica 
En la figura 6 se observan las absorbancias semanales obtenidas a $405 \mathrm{~nm}$ para IgG en los sueros de los grupos de estudio, contra la leucotoxina de Mannhemia haemolytica. No se observaron diferencias estadísticas significativas en el promedio de las absorbancias para el grupo $A$ y el grupo $B$ durante el estudio $(P>0.05)$; es a partir de la semana 3 que se observan diferencias entre $A$ y $B$ con respecto al grupo $C(P<0.05)$.

\section{DISCUSIÓN}

En este estudio, se utilizaron conejos como modelo biológico para observar el efecto de la suplementación con selenio y evaluar parte de la respuesta inmune humoral, contra Mannheimia haemolytica serotipo A2 y su leucotoxina; la cual afecta el tracto respiratorio de los rumiantes, causando neumonía y muerte de éstos.

El requerimiento de selenio es bajo para conejos, con alrededor de $0.05 \mathrm{mg} / \mathrm{kg}$ de alimento, para observar efectos benéficos en la productividad de estos animales (NRC, 1977; Papadomichelakis et al., 2017); sin embargo se ha observado que dosis de 0.2 $\mathrm{mg} / \mathrm{kg}$ de alimento mejoran la productividad en esta especie (Syvyk et al., 2018).

Para suplementar a los animales por vía parenteral,se utilizó una dosis de $0.025 \mathrm{mg} / \mathrm{kg}$ de peso vivo, recomendada por Ramírez-Bribiesca et al., 2004 para rumiantes, extrapolando esta dosis al peso metabólico de los conejos, para que ésta fuera la adecuada para la especie; así como para evitar intoxicaciones y muertes.

Se ha reportado que los niveles adecuados de selenio en sangre de conejos, van de 0.074 a 1.000 ppm (Puls,1988), esto depende de la dieta y la zona geográfica donde se encuentren. Después de la suplementación se observó que el grupo A tuvo un promedio de $0.972 \mu \mathrm{g} / \mathrm{g}$ de selenio en sangre para todo el estudio; mientras que los grupos B y C tuvieron en promedio de $0.595 \mu \mathrm{g} / \mathrm{g}$ de selenio en sangre para todo el experimento $(\mathrm{P}<0.05)$.

No se observaron deficiencias de selenio en sangre, los tres grupos mantuvieron niveles adecuados durante todo el experimento. Se ha observado que la suplementación con el mineral incrementa los niveles de selenio en sangre en vacas lecheras (Khalili, et al., 2020), cerdos (Cao et al., 2014), en pollos (Doaa et al., 2019), borregos (Ademi et al., 2017) y cabras (Ziaei, 2015), con efectos benéficos en la salud y la producción animal; sin embargo, un exceso del mineral en la dieta puede llegar a tener efectos negativos en la productividad con la consecuente intoxicación de los animales y la muerte (Żarczyñska et al., 2013).

Se observó que hubo variaciones en las concentraciones de selenio a lo largo del experimento para todos los grupos. En algunas semanas estos muestran un decremento en las concentraciones del mineral en sangre, particularmente el grupo A, en las semanas 2 y 4. Esto también se observó en cerdos suplementados con selenio y desafiados con diferentes antígenos (Falka et al., 2018), esto tal vez sea debido a la biodistribución del mineral en el organismo, para mantener el balance oxidativo frente a infecciones 0 desafíos con antígenos, a través de las selenoproteínas; ya que éstas participan en la 
regulación del estrés oxidativo, optimizando los procesos celulares para su correcto funcionamiento; incluidos aquellos involucrados en respuestas inmunes innatas y adaptativas (Dalgaarda et al., 2018). Sin embargo, esta síntesis está regulada por la disponibilidad del mineral en el organismo; una deficiencia de selenio tendrá impacto en el correcto funcionamiento de las células y por lo tanto de las respuestas inmunológicas (Howard et al., 2013; Seyedali et al., 2014).

Los monogástricos como los conejos, no presentan una deficiencia de selenio tan marcada como los rumiantes; los cuales son muy susceptibles a la deficiencia de este mineral, en especial en ovinos y caprinos. En estas especies la digestibilidad y absorción de este mineral a través de la dieta es muy baja (11-18\%); en comparación con los monogástricos (70-80\%), lo cual afecta su salud y productividad (Ghany y Tórtora-Pérez, 2010). Esta mayor susceptibilidad de los rumiantes se atribuye al ambiente retículoruminal, ya que parte del selenio ingerido es tomado por la microbiota, la cual lo utiliza para la síntesis de proteínas; o el propio ambiente ruminal reduce el mineral hasta formas no solubles (selenuros), los cuales no pueden ser absorbidos por el animal (Carbajal et al., 2013).

Al evaluar las absorbancias para IgG en suero, contra Mannheimia haemolytica serotipo A2, se encontró que el grupo A presentó una absorbancia promedio de $1.087 \mathrm{~nm}$, significativamente mayor que los grupos $\mathrm{B}$ y $\mathrm{C}$, los cuales promediaron $0.817 \mathrm{~nm}$ en el estudio $(P<0.05)$.

En otros trabajos se han observado resultados ambiguos con respecto a la suplementación de selenio, y su efecto sobre la respuesta inmune frente a desafíos con diferentes antígenos. Por un lado, se ha visto que la suplementación con selenio ha tenido un efecto positivo en pollos en la inducción de anticuerpos específicos para la vacuna contra el virus de la enfermedad infecciosa bursal (Shekaro et al., 2012). De la misma forma, se ha observado una mayor respuesta inmune mediada por anticuerpos contra Pasteurella multocida en ovejas suplementadas con $0.3 \mathrm{ppm}$ de selenio en la dieta (Kumar et al., 2009). En contraste, cuando se estudió la influencia del selenio en la inmunidad de los pollos de engorda mediante la suplementación a través del alimento en varias concentraciones $(0,100,200,300 \circ 400 \mu \mathrm{g} / \mathrm{kg}$ de dieta), no se encontró ningún efecto en la producción de anticuerpos específicos para la vacuna contra el virus de la enfermedad de Newcastle (Rao et al., 2013). Por otro lado, un estudio en caprinos donde se evaluó la respuesta inmune contra Mannheimia haemolytica, al evaluar IgG en suero, no se observaron diferencias significativas en los grupos de estudio los primeros 28 días del experimento; sin embargo, los grupos suplementados mostraron una concentración significativamente mayor de lgG a partir del día 28, después y hasta el final del experimento (Díaz-Sánchez et al., 2017). Por último, a pesar de que los estudios en conejos son limitados, se ha reportado que conejos raza California, suplementados con selenio, y desafiados contra glóbulos rojos de las ovejas (SRBC), los títulos de anticuerpos fueron más altos en comparación con el grupo control (Ebeid et al., 2013), como se observó en este estudio para el caso de Mannheimia haemolytica. 
Es probable que haya más interacciones que tengan que ver con la suplementación con selenio y la respuesta inmune a antígenos; como por ejemplo el estatus nutricional de los animales, la edad, disponibilidad de selenio en el organismo y el tipo de agente infeccioso o antígeno que haya a entrado al organismo animal; por eso tal vez se observan diferencias entre animales con respecto a la suplementación de selenio y la respuesta inmune (Hoffmann y Berry, 2008).

Cuando hay una infección, la producción de especies reactivas de oxígeno (ROS), participa en la activación y señalización de diversos sistemas endógenos (Vladimirov et al., 2009). Las células fagocíticas dependen de la producción de ROS para sus actividades bactericidas durante la inflamación, pero si este proceso no se controla a través de los antioxidantes como las selenoproteínas, los productos reactivos de oxígeno pueden inducir daños en el huésped como la lipoperoxidación celular (Lubos et al., 2011). Por último, se han visto que los efectos de la suplementación con selenio no afectarían necesariamente la concentración de anticuerpos de igual forma. Se pueden esperar diferentes efectos en las respuestas de anticuerpos dirigidas, contra antígenos $T$ dependientes frente a antígenos T independientes (Dalgaarda et al., 2018).

En lo que respecta a la evaluación de las absorbancias para lgG, contra la leucotoxina de Mannheimia haemolytica, no se encontraron diferencias entre los grupos A y B $(P>0.05)$; sin embargo, ambos grupos presentaron mayores absorbancias que el grupo $C(P>0.05)$, el cual no tuvo una respuesta al antígeno, tal como se esperaba en el estudio. Se sabe que la leucotoxina induce efectos biológicos negativos en los leucocitos rumiantes de una manera especie-específica. El conejo no es susceptible a este antígeno; sin embargo, podría tener efectos similares en el sistema inmune de éstos. En rumiantes induce la secreción y la liberación de péptidos quimiotácticos vasoactivos; así como aumenta el número de leucocitos disponibles en el lugar de la inflamación, en donde se producen depósitos fibrinosos. Este proceso ocasiona una neumonía fibrinopurulenta aguda. Cualquier oportunidad de respuesta inmune secundaria es interrumpida por la actividad de la leucotoxina, que previene la blastogénesis de los linfocitos y la destrucción de los mismos leucocitos (Jaramillo et al., 2009). Por último, Jaramillo en el 2000, logró la purificación de una adhesina, que fue capaz de aglutinar eritrocitos de conejo de manera específica, concluyendo que las adhesinas de Mannhemia haemolytica juegan un importantísimo papel en la infección; ésto puede sugerir el por qué hubo una respuesta de IgG más marcada a Mannhemia haemolytica serotipo A2, que a la leucotoxina para este estudio.

\section{CONCLUSIÓN}

Los animales que fueron suplementados tuvieron una mayor concentración de selenio en sangre. Al utilizar el conejo como modelo biológico para evaluar la respuesta antigénica, se encontró que la suplementación con selenio tuvo efectos positivos sobre la respuesta a los antígenos de Mannheimia haemolytica serotipo A2, obteniendo mayor absorbancia 
en los conejos suplementados, en comparación con los que no fueron suplementados. En cuanto a la respuesta al antígeno para la leucotoxina de Mannheimia haemolytica, no se encontraron diferencias entre los grupos estudiados.

\section{LITERATURA CITADA}

ADEMI A, Bernhoft A, Govasmark E, Bytyqi H, Sivertsen T, Singh BR. 2017. Selenium and other mineral concentrations in feed and sheep's blood in Kosovo. Transl. Anim. Sci. 1:97-107. https://doi.org/10.2527/tas2016.0010

AVERY JC, Hoffmann PR. 2018. Selenium, Selenoproteins, and Immunity. Nutrients. 10(9):1-20. https://doi.org/10.3390/nu10091203

CAMPOS GCM. 2015. El Impacto de los Micronutrientes en la inmunidad de los animales. Nutrición Animal Tropical. 9(1): 1-23. ISSN: 2215-3527. https://doi.org/10.15517/nat.v9i1.18778

CAO J, Guo F, Zhang L, Dong B, Gong L. 2014. Effects of dietary Selenomethionine supplementation on growth performance, antioxidant status, plasma selenium concentration, and immune function in weaning pigs. Journal of Animal Science and Biotechnology. 5:46. https://doi.org/10.1186/2049-1891-5-46

CARBAJAL HMA, Aquí QG, Díaz GC. 2013. Uso de selenio en ovinos. AbanicoVet. 3(1):44-54. ISSN 2007-4204. https://www.medigraphic.com/cgibin/new/resumen.cgi?IDARTICULO=44582

DALGAARDA ST, Briensb M, Engberga MR, Lauridsena C. 2018. The influence of selenium and selenoproteins on immune responses of poultry and pigs. Animal Feed Science and Technology. 238:73-83. https://doi.org/10.1016/j.anifeedsci.2018.01.020

DÍAZ-SÁNCHEZ VM, Rodríguez-Patiño G, Ramírez-Noguera P, Ramírez-Bribiesca JE, Morales-Álvarez JF, Revilla-Vázquez AL, López-Arellano R. 2017. Dose of selenium in goat kids and its effect on the antigenic response to Mannheimia haemolytica and oxidative stress. Small Ruminant Research. 153:171-174. http://dx.doi.org/10.1016/j.smallrumres.2017.06.005

DE LA ROSA RJL, Jaramillo-Arango CJ, Martínez-Maya JJ, Aguilar Romero F, Hernández-Castro R, Suarez-Güemes F, Trigo-Tavera F. 2012. Frecuencia de aislamientos de Mannheimia haemolytica y Pasteurella multocida en becerras con signos clínicos de enfermedad respiratoria, en un complejo lechero del estado de Hidalgo, México. Vet. Méx. 43:(1). https://www.researchgate.net/publication/286067163 
DOAA I, Asmaa TY, Safaa IK, Ahmed H, Haiam AM, Ahmed SA, Ghada IAEIR, Mohamed TE. 2019. Effect of Dietary Modulation of Selenium Form and Level on Performance, Tissue Retention, Quality of Frozen Stored Meat and Gene Expression of Antioxidant Status in Ross Broiler Chickens. Animals. 9:342. https://doi.org/10.3390/ani9060342

EBEID TA, Zeweil H, Basyony M, Badry H. 2012. The Impact of Incorporation of Organic Selenium Into Meat on Growth Performance, Antioxidative Status, and Immune Response in Growing Rabbits. Proceedings 10 th World Rabbit Congress. 861-864. https://www.researchgate.net/publication/224921998

FALKA M, Bernhoftb A, Framstadc T, Salbud B, Wisloffb H, Kortnere TM, Kristoffersenb BA, Oropeza MM. 2018. Effects of dietary sodium selenite and organic selenium sources on immune and inflammatory responses and selenium deposition in growing pigs. Journal of Trace Elements in Medicine and Biology. 50:527-536. https://doi.org/10.1016/j.jtemb.2018.03.003

GELDERMAN A, Clapper J. 2013. Effects of inorganic or organic selenium on immunoglobulins in swine. Journal of Animal Science and Biotechnology. 4:47. https://doi.org/10.1186/2049-1891-4-47

GHANY HAE, Tórtora-Pérez JL. 2010. The importance of selenium and the effects of its deficiency in animal health. Small Ruminant Research. 89:185-192. https://doi.org/10.1016/j.smallrumres.2009.12.042

GHANY-HEFNAWY AE, López-Arellano R, Revilla-Vázquez A, Ramírez-Bribiesca E, Tórtora-Pérez J. 2007. The relationship between fetal and maternal selenium concentrations in sheep and goats. Small Ruminant Research. 73:174-180. https://doi.org/10.1016/j.smallrumres.2007.01.020

HOFFMANN PR, Berry MJ. 2008. The influence of selenium on immune responses. $\mathrm{Mol}$ Nutr Food Res. 52(11): 1273-1280. https://doi.org/10.1002/mnfr.200700330

JARAMILLO ACJ, Trigo TFJ, Suárez GF. 2009. Mannheimiosis bovina: etiología, prevención y control. Vet. Méx. 40:(3). ISSN 0301-5092. http://www.scielo.org.mx/scielo.php?script=sci_arttext\&pid=S0301-50922009000300008

JARAMILLO L, Díaz F, Hernández P, Debray H, Trigo F, Mendoza G. 2000. Purification and characterization of an adhesin from Pasteurella haemolytica. Glycobiology.10:31-37. https://doi.org/10.1093/glycob/10.1.31 
KHALILI M, Chamani M, Amanlou H, Nikkhah A, Sadeghi AA, Dehkordi FK, Rafiei M, Shirani V. 2020. The effect of feeding inorganic and organic selenium sources on the hematological blood parameters, reproduction and health of dairy cows in the transition period. Acta Scientiarum. Animal Sciences. 42:45371. https://doi.org/10.4025/actascianimsci.v42i1.45371

KUMAR N, Garg AK, Dass RS, Chaturvedi VK, Mudgal V, Varshney VP. 2009. Selenium supplementation influences growth performance, antioxidant status and immune response in lambs. Anim. Feed Sci. Technol. (153):77-87. https://doi.org/10.1016/j.anifeedsci.2009.06.007

LUBOS E, Loscalzo J, Handy DE. 2011. Glutathione peroxidase-1 in health and disease: from molecular mechanisms to therapeutic opportunities. Antioxid. Redox Signal. 15:1957-1997. https://doi.org/10.1089/ars.2010.3586

MEHDI Y, Dufrasne I. 2016. Selenium in Cattle: A Review. Molecules. 21(545):1-14 http://doi.org/10.3390/molecules21040545

MORALES-ÁLVAREZ FJ, Jaramillo-Meza L, Oropeza-Vázquez Z, Tórtora-Pérez JL, Trigo-Tavera FJ, Espino-Rosas G. 1993. Evaluación experimental de un inmunógeno de Pateurella haemolytica en corderos. Vet. Méx. 24:97-105. http://www.medigraphic.com/cgi- bin/new/resumen.cgi?IDARTICULO=12524

NRC. 1977 Nutrient Requirements of Rabbits. National Academy of Sciences. National Research Council, Washington, USA. ISBN: 0-309-02607-5. https://www.nap.edu/catalog/35/nutrient-requirements-of-rabbits-second-revised-edition1977

PAPADOMICHELAKIS G, Zoidis E, Pappas AC, Mountzouris KC, Fegeros K. 2017. Effects of increasing dietary organic selenium levels on meat fatty acid composition and oxidative stability in growing rabbits. Meat Science. 131:132-138. https://doi.org/10.1016/j.meatsci.2017.05.006

PULS R. 1988. Mineral levels in animal health diagnostic data. Sherpa International, British Columbia, Canada. ISBN 0-9693429-0-X. https://www.cabdirect.org/cabdirect/abstract/19892283865

RADWIŃSKA J, Katarzyna Ż. 2014. Effects of mineral deficiency on the health of young ruminants. J. Elem. 19(3):915-928.

https://www.researchgate.net/publication/279013357_Effects_of_mineral_deficiency_on _the_health_of_young_Ruminants 
RAMÍREZ-BRIBIESCA E, Hernández-Camacho E, Hernández-Calva LM, Tórtora-Pérez JL. 2004. Efecto de un suplemento parenteral con selenito de sodio en la mortalidad de corderos y los valores hemáticos de selenio. Agrociencia. 38:43-51. ISSN: 1405-3195. https://www.researchgate.net/publication/242624660

RAO SVR, Prakash B, Raju MVLN, Panda AK, Poonam S, Murthy OK. 2013. Effect of supplementing organic selenium on performance, carcass traits, oxidative parameters and immune responses in commercial broiler chickens. Asian-Aust. J. Anim. Sci. 26:247252. https://doi.org/10.5713/ajas.2012.12299

SEYEDALI A, Berry MJ. 2014. Nonsense-mediated decay factors are involved in the regulation of selenoprotein mRNA levels during selenium deficiency. RNA Publ. RNA Soc. 20:1248-1256.

https://doi.org/10.1261/rna.043463.113

SHEKARO A, Oladele SB, Abdu PA, Ibrahim NDG. 2012. Effect of selenium on the susceptibility of vaccinated cockerels against infectious bursal disease. J. Vet. Adv. 2:573-578. ISSN: 2251-7685. https://doi.org/10.5455/japa.20150315015216

SYVYK TL, Dyachenko LS, Tytariova OM, Shulko OP, Osipenko OP, Pirova LV, Bilkevych VV. 2018. Productivity of rabbits and balance of selenium in their body by feeding different doses of selenium. Bulgarian Journal of Agricultural Science. 24:480-483. https://www.researchgate.net/publication/325763729

SOLANET JJ, Malena R, Estein SM, Estevao BSG, Paolicchi FA. 2011. Desarrollo de una prueba de ELISA para detectar anticuerpos en carneros vacunados o infectados con Corynebacterium pseudotuberculosis. Revista Argentina de Microbiología. 43(1):9-17. eISSN: 1851-7617. https://ri.conicet.gov.ar/handle/11336/70918

VLADIMIROV YA, Proskurnina EV. 2009. Free radicals and cell chemiluminescence. Biochem. Moscow. 74:1545-1566.

http://protein.bio.msu.ru/biokhimiya/contents/v74/full/74131545.html

ŻARCZYÑSKA K, Sobiech P, Radwiñska J, Rêkawek W. 2013. Effects of selenium on animal health. J. Elem. 18(2):329-340. https://doi.org/10.5601/jelem.2013.18.2.12

ZIAEI N. 2015. Effect of selenium and vitamin E supplementation on reproductive indices and biochemical metabolites in Raieni goats. Journal of Applied Animal Research. 43(4):426-430. http://dx.doi.org/10.1080/09712119.2014.980415 